\title{
Analytical solution of dynamic thermo elasticity problem for the FGM thick-walled sphere
}

\author{
Saeed Momeni Bashusqeh ${ }^{1}$, Amir Sharifi Miavaghi ${ }^{2}$, Masoud Ajri khameslou ${ }^{1}$

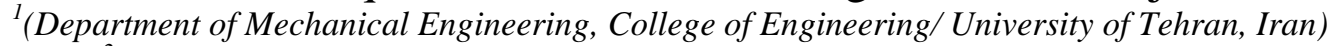 \\ ${ }^{2}$ (Department of Mechanical Engineering, Urmia University of Technology, Iran)
}

\begin{abstract}
Thermo-mechanical analysis of functionally graded hollow sphere subjected to time dependant mechanical and thermal boundary conditions is carried out analytically in this study. The material properties are assumed to vary non-linearly in the radial direction, and the Poisson's ratio is assumed constant. For thermal boundary conditions, temperature is prescribed on both surfaces whereas for mechanical boundary conditions tractions are prescribed on the boundaries. Obtaining the distribution of the temperature, the dynamical structural problem is solved and closed form solution is obtained for stress components.
\end{abstract}

Keywords: Thermoelasticity; FGM; hollow sphere; Hankel transform; thermal shock; wave propagation

\section{Introduction}

Functionally graded materials (FGMs) are being increasingly considered in various applications in the recent years. These materials have received considerable attention in many engineering applications since they were first reported in 1984 in Japan [1,2]. Because of Continuous change in the microstructure of FGMs may not be subjected to a mismatch of mechanical properties across the interface as a reinforced or laminated material does. As a result, these materials are able to withstand high-temperature gradients without structural failures. This feature is vital, especially in space and aeronautical applications. During the past years, many researchers have studied the characteristic behavior of FGMs under different loading conditions.

Analytical solutions have been done by Johnson and Mellor [3] for thick cylindrical vessels under pressure and temperature loading. Applying the Frobenius series method, Zimmerman and Lutz [4] found a way round the problem of the uniform heating of FG circular cylinder. They derived the exact solution for the problem of radially heated cylinder whose modulus of elasticity and thermal expansion coefficient vary linearly with radius. Another general analysis of one-dimensional steady-state thermal stresses in a hollow thick cylinder made of FGM was obtained by Eslami et al. [5]. They used a direct method to solve the heat conduction and Navier equations. Furthermore, the temperature distribution was assumed to be a function of radius. They used static formulation of thermo elasticity in their work In addition; Poultangari et al. [6] studied the steady state thermal and mechanical stresses in a FGM sphere under non-axisymmetric thermo-mechanical loads.

The quasi-static and static equations of thermoelasticity for FG cylinder were solved analytically in [78] and [9-12]. Wang et al. [13, 14] obtained the transient thermoelastic solutions of non-homogeneous and multilayered orthotropic hollow cylinders for axisymmetric plane strain problem by using the separation of variables method. It is noted that with the presence of inertial term, thermoelastic dynamic analysis becomes really complicated and difficult.

As for dynamic case: Cho et al. $[15,16]$ investigated the dynamic thermoelastic problem of a thick orthotropic cylindrical shell concerning the axial strain. Wang [17] studied the thermal shock effect in a hollow cylinder by means of finite Hankel transform and Laplace transform technique. Abd-alla[18] investigated the thermal stress in a transversely isotropic circular cylinder due to an instantaneous heat source by using the Laplace transform method. As a general rule, the effect of coupling term in the energy equation is small in many industrial applications and the distinction between coupled and uncoupled theories is negligible.

In this article Dynamic thermo elasticity problem of a pressurized sphere made of functionally graded material is solved analytically where material properties vary with radial position. What is new in this paper, in problems with a high loading rate, the inertia term in the equation of motion cannot be ignored and the problem is regarded as the dynamic thermo elasticity problem. Here, the dynamic thermo elasticity problem is solved using an innovative mathematical technique for thick sphere with FGM material. As a result of the analytical solution of the dynamic problem, thermal shock appeared in the results. Time dependant thermal and mechanical boundary conditions are assumed to act on the boundaries. For thermal boundary conditions, temperature is prescribed on both surfaces whereas for mechanical boundary conditions tractions are prescribed on the boundaries. In order to solve the problems, a finite Hankel transform is utilized to obtain the distribution of the temperature throughout the sphere and then this is used as input to solve the dynamical structural problem. A numerical example is considered as the case study; a hollow FG cylinder under thermal shock. Solving the problem, the results are shown graphically which in shock waves are obvious and the effect of 
material inhomogeneity is investigated too. The solution method used in this work has such a generality which is applicable for any other types of boundary conditions are prescribed just by altering the kernels of the transformations introduced in the subsequent sections.

\section{Formulation}

A hollow FGM sphere with the inner and the outer radii $a$ and $b$ is considered. The equations of uncoupled thermoelasticity at axisymmetric condition are

The energy equation:

$\frac{\partial^{2} T}{\partial r^{2}}+\left(\frac{k^{\prime}(r)}{k(r)}+\frac{2}{r}\right) \frac{\partial T}{\partial r}=\frac{\rho(r) c_{\circ}}{k(r)} \dot{T}$

The equation of motion:

$\frac{\partial \sigma_{r r}}{\partial r}+2 .\left(\frac{\sigma_{r r}-\sigma_{\theta \theta}}{r}\right)=\rho(r) \ddot{u}$

In which $\rho$ is the density, $k$ the thermal conduction coefficient, $c_{\circ}$ the specific heat and $\alpha$ the coefficient of thermal expansion. In the motion equation $\sigma_{r r}$ and $\sigma_{\theta \theta}$ are the radial and circumferential stress components, respectively. A dot over the quantity denotes its partial derivative with respect to time. In order to account for the changing material properties along the radius, a power law relationship [2] is used as follows

$$
\begin{aligned}
& k(r)=k_{\circ} r^{p} \\
& \rho(r)=\rho_{\circ} r^{p} \\
& E(r)=E_{\circ} r^{p} \\
& \alpha(r)=\alpha_{\circ} r^{p}
\end{aligned}
$$

Where $p$ is the power law indices of the material inhomogeneity. Constitutive law states that the relation between stress components, radial displacement component and temperature is as follows:

$$
\begin{aligned}
& \sigma_{r r}=\frac{E(r)}{(1+v)(1-2 v)}\left\{(1-v) \frac{\partial u}{\partial r}+2 v \frac{u}{r}-(1+v) \alpha(r) T\right\} \\
& \sigma_{\theta \theta}=\sigma_{\varphi \varphi}=\frac{E(r)}{(1+v)(1-2 v)}\left\{v \frac{\partial u}{\partial r}+v \frac{u}{r}-(1+v) \alpha(r) T\right\}
\end{aligned}
$$

The thermal boundary conditions of the sphere are considered as follows

$T(a, t)=f_{1}(t)$

$T(b, t)=f_{2}(t)$

At the inner and the outer surfaces are subjected to the traction boundary conditions; that is,

$$
\begin{aligned}
& \left.\frac{\partial u}{\partial r}\right|_{r=a}+k_{1} u(a, t)=B_{1}(t) \\
& \left.\frac{\partial u}{\partial r}\right|_{r=b}+k_{1} u(b, t)=B_{2}(t)
\end{aligned}
$$

All Initial conditions namely initial temperature, radial displacement and radial velocity are assumed to be zero.

Substituting Eqs. (2) and (3) into Eqs. (1a) and (1b) result in the following equations. 
$\frac{\partial^{2} T}{\partial r^{2}}+\frac{p+2}{r} \frac{\partial T}{\partial r}=\frac{\rho_{\mathrm{o}} c_{\mathrm{o}}}{k_{\mathrm{o}}} T$

$\frac{\partial^{2} u}{\partial r^{2}}+\frac{p+2}{r} \frac{\partial u}{\partial r}+\frac{2}{r^{2}}\left\{\frac{v p+v-1}{1-v}\right\} u-\frac{1+v}{1-v}\left\{\alpha \frac{\partial T}{\partial r}+\alpha_{\circ} p r^{p-1} T+\frac{p \alpha}{r} T\right\}$

$=\frac{\rho(r)(1+v)(1-2 v)}{E(r)(1-v)} \ddot{u}$

\section{Solution Of The Energy Equation}

Substituting equation (8) into Eq. (6a) gives the Eq. (8)

$\theta(r, t)=r^{\frac{p+1}{2}} T(r, t)$

$\frac{\partial^{2} \theta}{\partial r^{2}}+\frac{1}{r} \frac{\partial \theta}{\partial r}-\frac{\left(\frac{p+1}{2}\right)^{2}}{r^{2}} \theta=\frac{\rho_{\circ} c_{\circ}}{k_{\circ}} \dot{\theta}$

Rewriting the thermal boundary conditions we have

$$
\begin{aligned}
& \theta(a, t)=\sqrt{a^{p+1}} f_{1}(t) \\
& \theta(b, t)=\sqrt{b^{p+1}} f_{2}(t)
\end{aligned}
$$

Eq. (8) is Bessel type equation that may be solved using finite Hankel transform as defined in [19]

$$
H[X(r, t)]=\bar{X}(t)=\int_{a}^{b} r X(r, t) K(r) d r
$$

$$
K(r)
$$

is the kernel of the transformation. The choice of a proper kernel depends on the form of the governing equation and the boundary conditions of the problem. The kernel of the transformation should satisfy homogeneous boundary conditions, and hence can be considered as and should satisfy the corresponding homogeneous boundary conditions and hence can be considered as [19]

$$
H\left[\theta(r, t), \xi_{m}\right]=\bar{\theta}\left(t, \xi_{m}\right)=\int_{a}^{b} r \theta(r, t) K_{1}\left(r, \xi_{m}\right) d r
$$

As mentioned earlier, the kernel of the transformation must fulfill the homogeneous boundary conditions. Hence $K_{1}\left(r, \xi_{m}\right)$ are obtained [19]

$$
K_{1}\left(r, \xi_{m}\right)=Y_{(p+1) / 2}\left(\xi_{m} a\right) J_{(p+1) / 2}\left(\xi_{m} r\right)-J_{(p+1) / 2}\left(\xi_{m} a\right) Y_{(p+1) / 2}\left(\xi_{m} r\right)
$$

$\xi_{m}$ Is the transform parameter and they are the positive roots of the following equation

$$
J_{(p+1) / 2}\left(\xi_{m} a\right) Y_{(p+1) / 2}\left(\xi_{m} b\right)-J_{(p+1) / 2}\left(\xi_{m} b\right) Y_{(p+1) / 2}\left(\xi_{m} a\right)=0
$$

The Henkel inverse transform is defined as

$$
H^{-1}\left[\bar{\theta}\left(\xi_{m}, t\right) ; r\right]=\theta(r, t)=\sum_{m=1}^{\infty} a_{m} \bar{\theta}\left(\xi_{m}, t\right) K_{1}\left(r, \xi_{m}\right)
$$

Using orthogonal property of Bessel functions, we have 
$a_{m}=\frac{1}{\int_{a}^{b} r K_{1}^{2}\left(r, \xi_{m}\right) d r}$

Then the $a_{m}$ can be obtained

$a_{m}=\frac{\pi^{2}}{2} \frac{\xi_{m}^{2} J_{(p+1) / 2}^{2}\left(\xi_{m} b\right)}{J_{(p+1) / 2}^{2}\left(\xi_{m} a\right)-J_{(p+1) / 2}^{2}\left(\xi_{m} b\right)}$

Applying the Hankel transform to the heat conduction equation, one needs the following relationship to express the Hankel transform of the derivatives of the temperature to the Hankel transform of the temperature by itself [19]:

$$
\begin{aligned}
& H\left[\frac{\partial^{2} \theta}{\partial r^{2}}+\frac{1}{r} \frac{\partial \theta}{\partial r}-\frac{\left(\frac{p}{2}\right)^{2}}{r^{2}} \theta\right]=\frac{2 J_{(p+1) / 2}\left(\xi_{m} a\right)}{\pi J_{(p+1) / 2}\left(\xi_{m} b\right)} \theta(b)-\frac{2}{\pi} \theta(a)-\xi_{m}^{2} \bar{\theta}( \\
& =\frac{\rho_{\circ} c_{\circ}}{k_{\circ}} \dot{\bar{\theta}}\left(t, \xi_{m}\right)
\end{aligned}
$$

Substituting Eqs. (9a) and (9b) into the above equation result in the following equation

$-\xi_{m}^{2} \bar{\theta}+\frac{2 J_{(p+1) / 2}\left(\xi_{m} a\right)}{\pi J_{(p+1) / 2}\left(\xi_{m} b\right)} \sqrt{b^{p+1}} f_{2}(t)-\frac{2}{\pi} \sqrt{a^{p+1}} f_{1}(t)=\frac{\rho_{\circ} c_{\circ}}{k_{\circ}} \dot{\bar{\theta}}$

Which its solution gives $\bar{\theta}\left(t, \xi_{m}\right)$ as

$\bar{\theta}\left(t, \xi_{m}\right)=\int_{0}^{t} A_{1}(\tau) e^{-\frac{k_{\circ}}{\rho_{\mathrm{o}} c_{\mathrm{o}}} \xi_{m}^{2}(t-\tau)} d \tau$

$A_{1}(t)=\frac{2 k_{\circ}}{\pi \rho_{\circ} c_{\circ}}\left[\frac{J_{(p+1) / 2}\left(\xi_{m} a\right)}{J_{(p+1) / 2}\left(\xi_{m} b\right)} \sqrt{b^{p+1}} f_{2}(t)-\sqrt{a^{p+1}} f_{1}(t)\right]$

Applying inverse transform into Equation (15) the temperature distribution is obtained as

$$
\begin{aligned}
& T(r, t)=\frac{\pi^{2}}{2 \sqrt{r^{p+1}}} \sum_{\xi_{m}} \frac{\xi_{m}^{2} J_{(p+1) / 2}^{2}\left(\xi_{m} b\right) \bar{\theta}\left(\xi_{m}, t\right)}{J_{(p+1) / 2}^{2}\left(\xi_{m} a\right)-J_{p / 2}^{2}\left(\xi_{m} b\right)} \\
& \cdot\left[J_{(p+1) / 2}\left(\xi_{m} r\right) Y_{(p+1) / 2}\left(\xi_{m} a\right)-J_{(p+1) / 2}\left(\xi_{m} a\right) Y_{(p+1) / 2}\left(\xi_{m} r\right)\right]
\end{aligned}
$$

\section{Solution Of The Equation Of Motion}

Solution of the equation of motion is not as easily as of the energy equation. Introducing $w=r^{\frac{p+1}{2}} u$

using above equation Eq. (6b) can be rewritten as 
$\frac{\partial^{2} w}{\partial r^{2}}+\frac{1}{r} \frac{\partial w}{\partial r}-\frac{\eta^{2}}{r^{2}} w-\frac{1+v}{1-v}\left\{\alpha \frac{\partial \theta}{\partial r}+\left[\alpha_{\circ} p r^{p-1}+\frac{(p+1) \alpha}{2 r}\right] \theta\right\}=\gamma^{2} \ddot{w}$

In which

$\eta^{2}=\left(\frac{p+1}{2}\right)^{2}-\frac{2 v p}{1-v}+2$

$\gamma^{2}=\frac{\rho(r)(1+v)(1-2 v)}{E(r)(1-v)}$

Applying Eq. (16) into Eqs. (6a) and (6b) i.e. the structural boundary conditions we have

$\left.\frac{\partial w}{\partial r}\right|_{r=a}+k_{1}^{\prime} w(a, t)=B_{1}^{\prime}(t)$

$\left.\frac{\partial w}{\partial r}\right|_{r=b}+k_{2}^{\prime} w(b, t)=B_{2}^{\prime}(t)$

Where

$k_{1}^{\prime}=k_{1}-\frac{p+1}{2 a}$

$k_{2}^{\prime}=k_{2}-\frac{p+1}{2 b}$

$B_{1}^{\prime}=\sqrt{a^{p+1}} B_{1}(t)$

$B_{2}^{\prime}=\sqrt{b^{p+1}} B_{2}(t)$

Field variable $w(r, t)$ is resolved into two variables $w_{1}(r, t)$ and $w_{2}(r, t)$ such that

$\frac{\partial^{2} w_{1}}{\partial r^{2}}+\frac{1}{r} \frac{\partial w_{1}}{\partial r}-\frac{\eta^{2}}{r^{2}} w_{1}=\gamma^{2} \ddot{w_{1}}$

$\left.\frac{\partial w_{1}}{\partial r}\right|_{r=a}+k_{1}^{\prime} w_{1}(a, t)=B_{1}^{\prime}(t)$

$\left.\frac{\partial w_{2}}{\partial r}\right|_{r=b}+k_{2}^{\prime} w_{2}(b, t)=B_{2}^{\prime}(t)$

And

$\frac{\partial^{2} w_{2}}{\partial r^{2}}+\frac{1}{r} \frac{\partial w_{2}}{\partial r}-\frac{\eta^{2}}{r^{2}} w_{2}-\frac{1+v}{1-v}\left\{\alpha \frac{\partial \theta}{\partial r}+\left[\alpha_{\circ} p r^{p-1}+\frac{(p+1) \alpha}{2 r}\right] \theta\right\}=\gamma^{2} \ddot{w_{2}}$

$\left.\frac{\partial w_{2}}{\partial r}\right|_{r=a}+k_{1}^{\prime} w_{2}(a, t)=0$

$\left.\frac{\partial w_{2}}{\partial r}\right|_{r=b}+k_{2}^{\prime} w_{2}(b, t)=0$

Eq. (20) is Bessel equation which is solved using Hankel transform. The solution procedure is like that 
followed in the previous section but with some differences which arises because boundary conditions are of different types. In section 3 boundary conditions were of Dirichlet type whereas in structural problem Cauchy type boundary conditions were prescribed on the boundaries. As mentioned in the previous section, the kernel of the transformation must satisfy homogeneous boundary conditions means that

$$
\begin{aligned}
& \left.\frac{\partial K_{2}}{\partial r}\right|_{r=a}+k_{1}^{\prime} K_{2}(a, t)=0 \\
& \left.\frac{\partial K_{2}}{\partial r}\right|_{r=b}+k_{2}^{\prime} K_{2}(b, t)=0
\end{aligned}
$$

[19]

The kernel of the transformation with considering the above equations may be chosen as the following

$$
\begin{aligned}
& K_{2}\left(r, \lambda_{n}\right)=\left[\left.\frac{d Y_{\eta}\left(\lambda_{n} r\right)}{d r}\right|_{r=a}+k_{1}^{\prime} Y_{\eta}\left(\lambda_{n} a\right)\right] J_{\eta}\left(\lambda_{n} r\right) \\
& -\left[\left.\frac{d J_{\eta}\left(\lambda_{n} r\right)}{d r}\right|_{r=a}+k_{1}^{\prime} J_{\eta}\left(\lambda_{n} a\right)\right] Y_{\eta}\left(\lambda_{n} r\right)
\end{aligned}
$$

$\lambda_{n}$ are the positive roots of the following equation

$$
\begin{aligned}
& {\left[\left.\frac{d Y_{\eta}\left(\lambda_{n} r\right)}{d r}\right|_{r=a}+k_{1}^{\prime} Y_{\eta}\left(\lambda_{n} a\right)\right]\left[\left.\frac{d J_{\eta}\left(\lambda_{n} r\right)}{d r}\right|_{r=b}+k_{2}^{\prime} J_{\eta}\left(\lambda_{n} b\right)\right]=} \\
& {\left[\left.\frac{d Y_{\eta}\left(\lambda_{n} r\right)}{d r}\right|_{r=b}+k_{2}^{\prime} Y_{\eta}\left(\lambda_{n} b\right)\right]\left[\left.\frac{d J_{\eta}\left(\lambda_{n} r\right)}{d r}\right|_{r=a}+k_{1}^{\prime} J_{\eta}\left(\lambda_{n} a\right)\right]}
\end{aligned}
$$
we have

Using Hankel transformation in Eq. (20a) and utilizing Bessel functions identities as was done in [19],

$$
-\lambda_{n}^{2} \bar{w}_{1}+\frac{2 E_{1}}{\pi E_{2}} B_{2}^{\prime}(t)-\frac{2}{\pi} B_{1}^{\prime}(t)=\gamma^{2} \ddot{w}_{1}
$$

In Which

$$
\begin{aligned}
& E_{1}=\left[\left.\frac{d J_{\eta}\left(\lambda_{n} r\right)}{d r}\right|_{r=a}+k_{1}^{\prime} J_{\eta}\left(\lambda_{n} a\right)\right] \\
& E_{2}=\left[\left.\frac{d J_{\eta}\left(\lambda_{n} r\right)}{d r}\right|_{r=b}+k_{2}^{\prime} J_{\eta}\left(\lambda_{n} b\right)\right]
\end{aligned}
$$

Solution of the above ordinary differential equation, Eq. (24a), gives $\bar{w}_{1}(r, t)$ as $\bar{w}_{1}\left(t, \lambda_{n}\right)=\frac{\gamma}{\lambda_{n}} \int_{0}^{t} A_{2}(\tau) \sin \left(\frac{\lambda_{n}}{\gamma}(t-\tau)\right) d \tau$

where

$$
A_{2}(t)=\frac{2}{\pi \gamma^{2}}\left[\frac{E_{1}}{E_{2}} B_{2}^{\prime}(t)-B_{1}^{\prime}(t)\right]
$$


Then $w_{1}(r, t)$ is obtained using the inverse Hankel transform which is defined as

$H^{-1}\left[\bar{w}_{1}\left(\lambda_{n}, t\right) ; r\right]=w(r, t)=\sum_{n=1}^{\infty} b_{n} \bar{w}_{1}\left(\lambda_{n}, t\right) K_{2}\left(r, \lambda_{n}\right)$

In which [19]

$$
\begin{aligned}
& b_{n}=\frac{1}{\int_{a}^{b} r K_{2}^{2}\left(r, \lambda_{n}\right) d r} \\
& =\frac{\pi^{2} \lambda_{n}^{2} E_{2}^{2}}{2\left\{\left(k_{2}^{\prime 2}+\lambda_{n}^{2}\left[1-\left(\frac{\eta}{\lambda_{n} b}\right)^{2}\right]\right) E_{1}^{2}-\left(k_{1}^{\prime 2}+\lambda_{n}^{2}\left[1-\left(\frac{\eta}{\lambda_{n} a}\right)^{2}\right]\right) E_{2}^{2}\right\}}
\end{aligned}
$$

$w_{2}(r, t)$ is considered to be multiplying of two function $S(t)$ and $K_{2}\left(r, \lambda_{n}\right)$

$w_{2}(r, t)=S(t) K_{2}\left(r, \lambda_{n}\right)$

In which $S(t)$ is unknown function of time. Since boundary conditions of Eq. (21a), Eqs. (21b) and (21b), are homogeneous and also the kernel of the transformation satisfy homogeneous boundary conditions, the general form considered above is valid. Inserting Eq. (27) into Eq. (21a) yields

$$
K_{2}\left(r, \lambda_{n}\right)\left[\gamma^{2} \ddot{S}+\lambda_{n}^{2} S(t)\right]=-\frac{1+v}{1-v}\left\{\alpha \frac{\partial \theta}{\partial r}+\left[\alpha_{\mathrm{o}} p r^{p-1}+\frac{(p+1) \alpha}{2 r}\right] \theta\right\}
$$

Orthogonal property of Bessel functions are employed to compute $S(t)$ as follows

$\int_{a}^{b} r K_{2}\left(\lambda_{n}, r\right) K_{2}\left(\lambda_{q}, r\right) d r=N_{n} \delta_{n q}$

$\delta_{n q}$ is the Kronecker delta and

$N_{n}=\frac{1}{2 \lambda_{n}^{2}} \times$

$\left\{\left.b^{2} \frac{d K_{2}}{d r}\right|_{r=b} ^{2}-\left.a^{2} \frac{d K_{2}}{d r}\right|_{r=a} ^{2}+\lambda_{n}^{2}\left\{b^{2} K_{2}^{2}(b)-a^{2} K_{2}^{2}(a)\right\}-\eta^{2}\left\{b^{2} K_{2}^{2}(b)-a^{2} K_{2}^{2}\right.\right.$

Multiplying both sides of Eq. (28) by $r K_{2}\left(r, \lambda_{n}\right)$ and integrating over the thickness of the cylinder, we have

$\ddot{S}+\left(\frac{\lambda_{n}}{\gamma}\right)^{2} S(t)=-\frac{1+v}{1-v} \frac{\int_{a}^{b} r K_{2}\left\{\alpha \frac{\partial \theta}{\partial r}+\left[\alpha_{\circ} p r^{p-1}+\frac{(p+1) \alpha}{2 r}\right] \theta\right\} d r}{\gamma^{2} N_{n}}$

Substituting Eq. (11) into the above equation gives the following ordinary equation

$\ddot{S}+\left(\frac{\lambda_{n}}{\gamma}\right)^{2} S(t)=-\frac{a_{m}(1+v) \bar{\theta}}{\gamma^{2}(1-v)} \frac{\int_{a}^{b} r K_{2}\left\{\alpha \frac{\partial K_{1}}{\partial r}+\left[\alpha_{\circ} p r^{p-1}+\frac{(p+1) \alpha}{2 r}\right]\right.}{N_{n}}$ 
Solving above equation the $S(t)$ can be obtained as follows

$S(t)=\frac{\gamma}{\lambda_{n}} \int_{0}^{t} A_{3}(\tau) \sin \left(\frac{\lambda_{n}}{\gamma}(t-\tau)\right) d \tau$

$A_{3}(t)=-\frac{a_{m}(1+v) \bar{\theta}}{\gamma^{2}(1-v)} \frac{\int_{a}^{b} r K_{2}\left\{\alpha \frac{\partial K_{1}}{\partial r}+\left[\alpha_{\circ} p r^{p-1}+\frac{(p+1) \alpha}{2 r}\right] K_{1}\right\} d r}{\int_{a}^{b} r K_{2}^{2} d r}$

Then $u(r, t)$ can be obtained

$u(r, t)=\frac{1}{\sqrt{r^{p+1}}} \sum_{\lambda_{n}}^{\infty} \sum_{\xi_{m}}\left[b_{n} \bar{w}_{1}(t)+S(t)\right] K_{2}(r)$

are:

Computing $T(r, t)$ and $u(r, t)$, stress components can be computed using Eqs. (3a) and (3b) which

$$
\begin{aligned}
& \sigma_{r r}=\frac{1}{\sqrt{r^{p+1}}} \sum_{\lambda_{n}}^{\infty} \sum_{\xi_{m}}^{\infty}\left[b_{n} \bar{w}_{1}(t)+S(t)\right]\left\{(1-v) \frac{\partial}{\partial r}\left[\frac{K_{2}(r)}{\sqrt{r^{p+1}}}\right]+\frac{2 v}{r \sqrt{r^{p+1}}} K\right. \\
& -\frac{E \alpha}{(1-2 v) \sqrt{r^{p+1}}} T(r, t) \\
& \sigma_{\theta \theta}=\frac{1}{\sqrt{r^{p+1}}} \sum_{\lambda_{n}}^{\infty} \sum_{\xi_{m}}^{\infty}\left[b_{n} \bar{w}_{1}(t)+S(t)\right]\left\{v \frac{\partial}{\partial r}\left[\frac{K_{2}(r)}{\sqrt{r^{p+1}}}\right]+\frac{1}{r \sqrt{r^{p+1}}} K_{2}(r)\right. \\
& -\frac{E \alpha}{(1-2 v) \sqrt{r^{p+1}}} T(r, t)
\end{aligned}
$$

\section{Conclusion}

The analytical solution presented in the previous section was applied to a thick hollow sphere of inner radius $a=1 \mathrm{~m}$, and outer radius of $b=2 \mathrm{~m}$. The hollow sphere assumed to be traction free at inner and outer surfaces. The thermal boundary conditions are as follows:

$$
\begin{aligned}
& T(a, t)=T_{0} \\
& T(b, t)=0
\end{aligned}
$$

The material properties of sphere are shown in Table.1.

Solving the problem, the results are shown graphically which in shock waves are obvious and the effect of material inhomogeneity is investigated too. The temperature distribution through the wall of sphere has shown in Fig.1. It can be seen that with increasing the time the temperature distribution reaches to steady state one.

The distribution of hoop and radial stress at $r=1.5 \mathrm{~m}$ are plotted versus time in Figs.2, 3.Nondimensional stress components have been used in these figures as follows:

$$
\sigma_{1}=\frac{(1-2 v) \sigma_{r r}}{E \alpha \theta_{0}}
$$




$$
\sigma_{2}=\frac{(1-2 v) \sigma_{\theta \theta}}{E \alpha \theta_{0}}
$$

The thermal shock phenomena can be seen in these Figs which relate to the hyperbolic nature of equation (1b).the shock waves for all p after $8 \times 10^{-5}$ second reach to $r=1.5 \mathrm{~m}$. It is worth mentioning that when the tensile wave reaches any radial position, tangential stress components also become suddenly tensile due to the resistance exerted by the medium; the wave decays gradually with time. These figures also shows that the amplitude of both radial and hoop stress become larger with increasing power law index of FGM sphere.

The History of radial displacement at mentioned location are plotted for different values of $p$ are plotted at Fig.4. The radial displacement value become greater with increasing time up to $8 \times 10^{-5}$ second after this time reduce and its value become negative. Also greater ${ }^{p}$ result in larger radial displacement values at $15 \times 10^{-5}$ second.

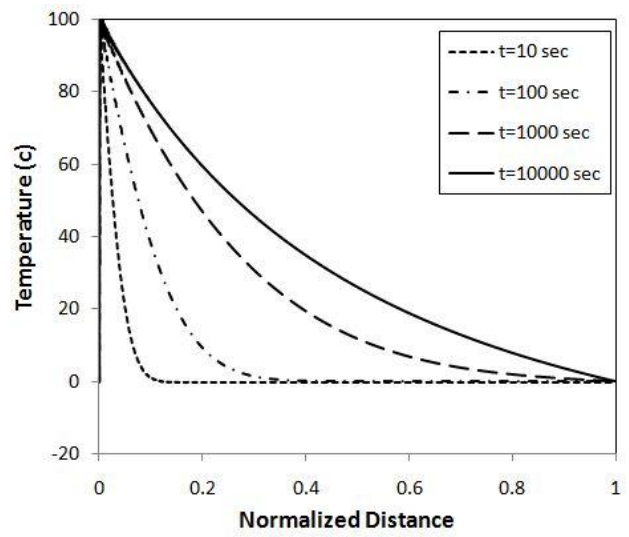

Figure 1. Time-dependent temperature distribution versus the radial distance

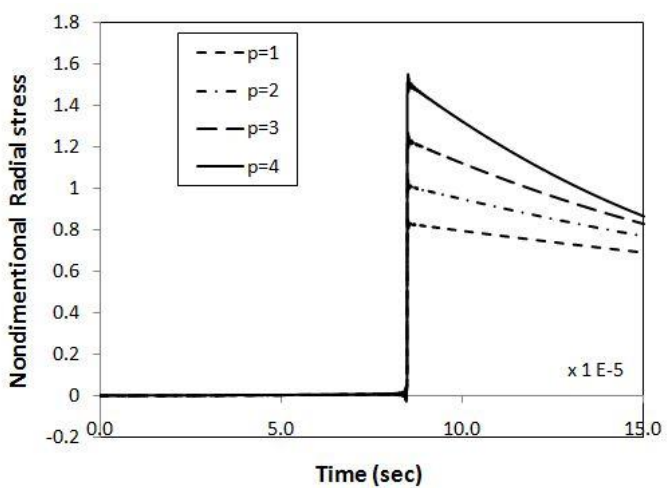

Figure 2. History of non-dimensional radial stress

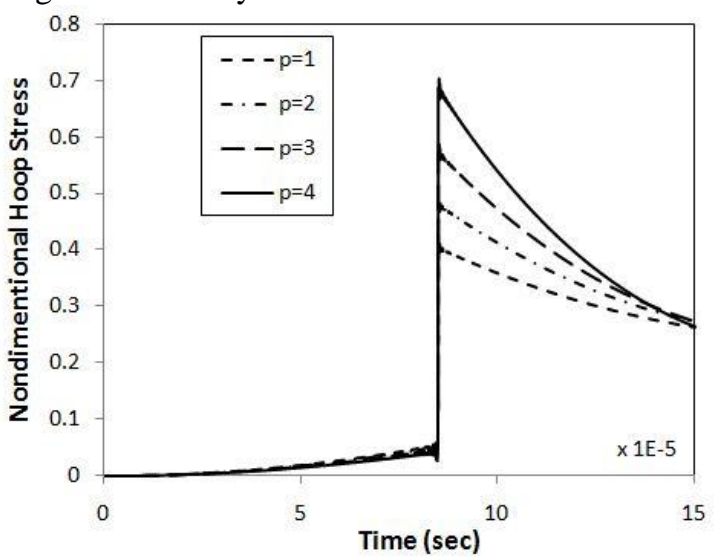

Figure 3. History of non-dimensional hoop stress 


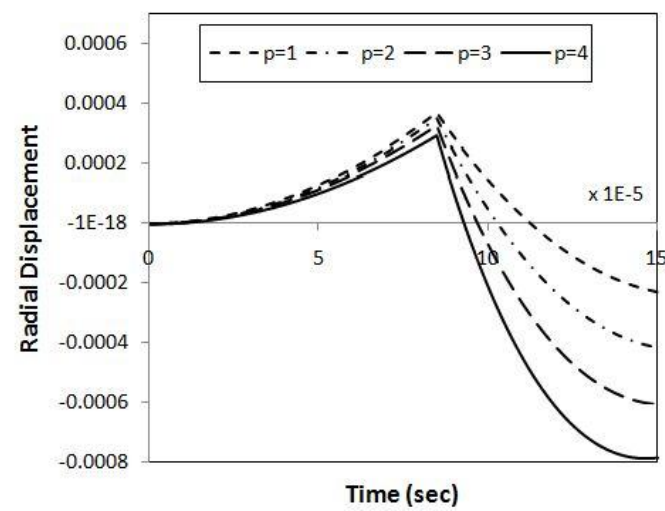

Figure 4. History of radial displacement

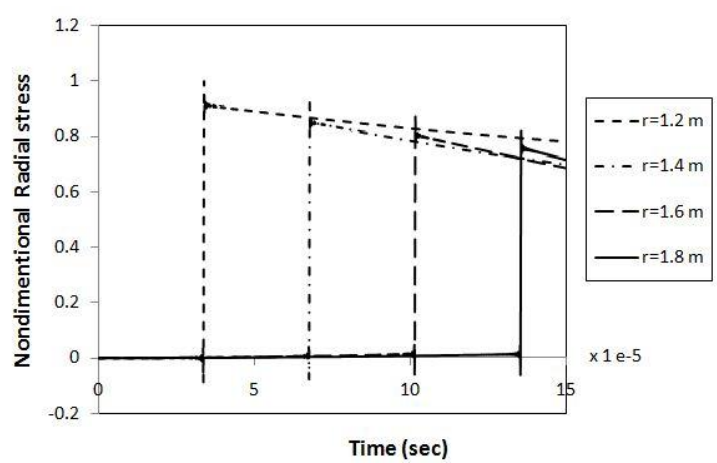

Figure 5. History of non-dimensional radial stress at different $r$

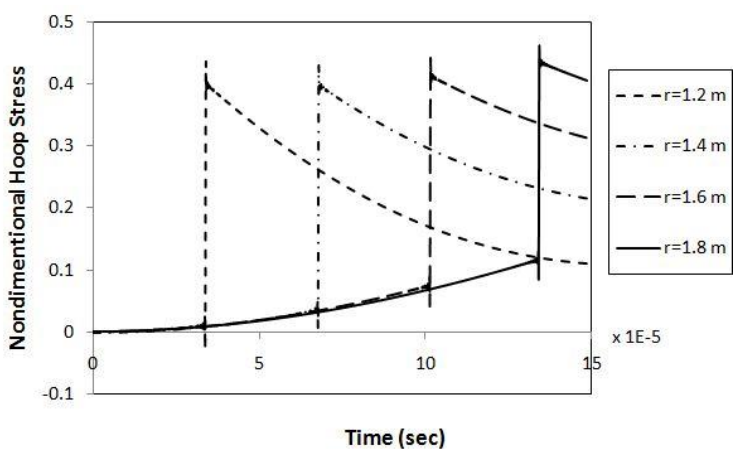

Figure 6. History of non-dimensional hoop stress at different $r$

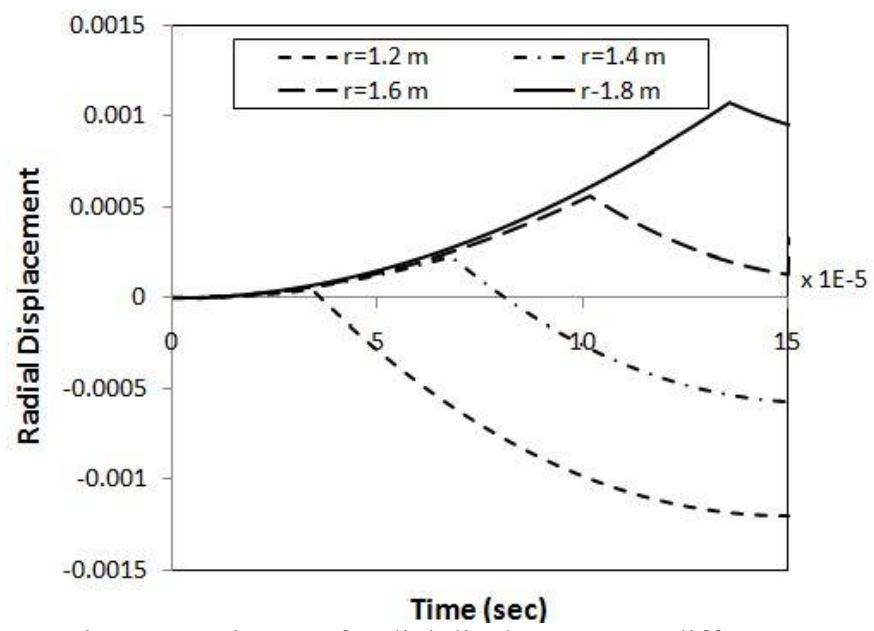

Figure 7. History of radial displacement at different $r$ 
Table.1. material properties

\begin{tabular}{llllll}
\hline$v=0.3$ & & $E=70 G P a$ & $\rho=2707$ & $\mathrm{~kg} / \mathrm{m}^{3}$ \\
$k=204$ & $W / \mathrm{mk}^{3}$ & $c=903$ & $\mathrm{~J} / \mathrm{kgK}$ & $\alpha=23 \times 10^{-6}$ & $1 / \mathrm{K}$ \\
$p=1,2,3,4$ & & & & \\
\hline
\end{tabular}

The distribution of hoop and radial stress at $r=1.2,1.4,1.6,1.8 m$ are plotted versus time in Figs.5, 7.The spherical dilatation wave propagates outward from the inner boundary and at different times reach to selected location. Also the amplitude of radial stress is greater than hoop stress. The wave decays gradually with time for both non-dimentiontional radial and hoop stresses.

History of radial displacement at selected position is plotted at Fig.7. As the figure shows that at outer radius the radial displacement for all times is greater than inner ones. As the time increase the radial displacement become more positive and then decrease and become negative

\section{Acknowledgements}

Authors would like to thank Savalan Demir Yol Company for their financial supports.

\section{References}

[1] Yamanouchi, M., Koizumi, M., Shiota, I. Proceedings of the First International Symposium on Functionally Gradient Materials, Japan, 1990, 273-281.

[2] Koizumu, M. The concept of FGM, ceramic transactions. Funct. Grad. Mater. 34, 1993, 3-10.

[3] Johnson, W., Mellor, P.B. Engineering Plasticity (Ellis Harwood Ltd, Chichester, 1983)

[4] Zimmerman, R.W., Lutz, M.P. Thermal stresses and thermal expansion in a uniformly heated functionally graded cylinder.J. Therm. Stress 22, 1999 177-188.

[5] Jabbari, M., Sohrabpour, S., Eslami, M.R. Mechanical and thermal stresses in a functionally graded hollow cylinder due to radially symmetric loads. Int. J. Press. Vessel. Pipi. 79, 2002, 493-497

[6] S.M. Hosseini, M. Akhlaghi. Analytical solution in transient thermoelasticity of functionally graded thick hollow cylinders (Pseudodynamic analysis). Math. Methods Appl. Sci. 32, 2009 , 2019-2034.

[7] S.M. Hosseini, M. Akhlaghi. Analytical solution in transient thermoelasticity of functionally graded thick hollow cylinders (Pseudodynamic analysis). Math. Methods Appl. Sci. 32, 2009, 2019-2034.

[8] M. Jabbari, A. Bahtui and M.R. Eslami. Axisymmetric mechanical and thermal stresses in thick short length FGM cylinders. Int. J. Pres. Ves. Pip. 86, 2009, 296-306,.

[9] M. Jabbari, A. Bahtui and M.R. Eslami. Axisymmetric mechanical and thermal stresses in thick long FGM cylinder. J. Therm. Stresses. 29, 2006, 643-663.

[10] Y. Ootao and Y. Tanigawa. Transient thermoelastic analysis for a functionally graded hollow cylinder. J. Therm. Stresses. 29, 2006, 1031-1046.

[11] Z.S. Shao.: Mechanical and thermal stresses of a functionally graded circular hollow cylinder with finite length. Int. J. Pres. Ves. Pip. $82,155-163(2005)$

[12] M. Ruhi, A. Angoshtari and R. Naghdabadi,. Thermoelastic analysis of thick-walled finite-length cylinders of functionally graded materials. J. Therm. Stresses, 2005, 28391-408.

[13] Ding HJ, Wang HM, Chen WQ. A solution of a non-homogeneous orthotropic cylindrical shell for axisymmetric plane strain dynamic thermoelastic problems. J Sound Vib ,.263, 2003, 815-829.

[14] Wang HM, Ding HJ. Transient thermoelastic solution of a multilayered orthotropic hollow cylinder for axisymmetric problems. J Therm Stresses .27, 2004, 1169-1185

[15] Cho H, Kardomateas GA, Valle CS. Elastodynamic solution for the thermal shock stresses in an orthotropic thick cylindrical shell. ASME J Appl Mech. 65(1), 1998, 184-192.

[16] Cho H, Kardomateas GA. Thermal shock stresses due to heat convection at a bounding surface in a thick orthotropic cylindrical shell. Int J Solids Struct. 38, 2001, 2769-2788.

[17] Wang X. Thermal shock in a hollow cylinder caused by rapid arbitrary heating. J Sound Vib . 183, 1995, 899-906.

[18] Abd-alla AM. Thermal stress in a transversely isotropic circular cylinder due to an instantaneous heat source. Appl Math Comput $.68,1995,113-124$

[19] G. Cinelli. An extension of the finite Hankel transform and applications, Int. J. Eng. Sci. 3, 1965, 539-559. 